\title{
Impact of Dynamic Path Loss Models in an Urban Obstacle Aware Ad Hoc Network Environment
}

\author{
Kashif Amjad, ${ }^{1}$ Muhammad Ali, ${ }^{2}$ Sohail Jabbar, ${ }^{1,3}$ Majid Hussain, ${ }^{3}$ \\ Seungmin Rho, ${ }^{4}$ and Mucheol Kim ${ }^{4}$ \\ ${ }^{1}$ Department of Computer Science, Bahria University, Islamabad 44000, Pakistan \\ ${ }^{2}$ Department of Electrical Engineering, COMSATS Institute of Information Technology, Islamabad 44000, Pakistan \\ ${ }^{3}$ Department of Computer Science, COMSATS Institute of Information Technology, Sahiwal 57000, Pakistan \\ ${ }^{4}$ Department of Multimedia, Sungkyul University, Anyang-si 430-742, Republic of Korea
}

Correspondence should be addressed to Mucheol Kim; mucheol.kim@gmail.com

Received 2 December 2014; Accepted 3 February 2015

Academic Editor: Yasuko Y. Maruo

Copyright (C) 2015 Kashif Amjad et al. This is an open access article distributed under the Creative Commons Attribution License, which permits unrestricted use, distribution, and reproduction in any medium, provided the original work is properly cited.

\begin{abstract}
This study highlights the importance of the physical layer and its impact on network performance in Mobile Ad Hoc Networks (MANETs). This was demonstrated by simulating various MANET scenarios using Network Simulator-2 (NS-2) with enhanced capability by adding propagation loss models (e.g., modified Two-Ray Ground model, ITU Line of Sight and Nonline of Sight (ITU-LoS and NLoS) model into street canyons and combined path loss and shadowing model (C-Shadowing)). The simulation results were then compared with the original Two-Ray Ground (TRG) model already available into NS-2. The scenario primarily simulated was that of a mobile environment using Random Way Point (RWP) mobility model with a variable number of obstacles in the simulation field (such as buildings, etc., causing variable attenuation) in order to analyze the extent of communication losses in various propagation loss models. Performance of the Ad Hoc On-demand Distance Vector (AODV) routing protocol was also analyzed in an ad hoc environment with 20 nodes.
\end{abstract}

\section{Introduction}

Mobile Ad Hoc Networks are formed by a collection of mobile nodes that can establish wireless communication links among each other without any infrastructure. This feature gives such networks a distinguished edge over other networks (such as GSM, UMTS, CDMA, and LTE). However it also brings new challenges and performance compromises (in terms of reliability, quality of service, scalability, etc.). Up until today, the majority of published research in MANETs has used simulation tools as a prime mean for performance analysis. This is primarily due to the high cost involved in realization of real ad hoc test beds. NS-2 [1] is the most popular simulation tool in MANETs researcher community [2]. This tool accommodates various routing, mobility, and propagation features key to analyze the performance of MANETs. However, this tool considers flat terrain for simulation and does not accommodate geographical features of the simulation field that may affect the received signal strength at the receiver. In [3], the author has introduced specialized mobility models, which restrict the mobility of nodes due to obstacles in the simulation area and some variations have been suggested in [4] for NS-2 environment. However the focus of their work is mainly the mobility aspect of the nodes. Contrarily, a vast majority of simulation studies have used simplistic radio propagation models [5] such as Two-Ray Ground (TRG) for performance analysis of MANETs routing strategies, which results in more optimistic rather than realistic network performance. This study accommodates several new propagation models (i.e., ITU Line of Sight (LoS) and Nonline of Sight (NLoS) path loss models in street canyons [6], and combined shadowingpath loss model [7] along with modified-TRG model) in an obstacle aware mobility environment. By varying the number of obstacles in the simulation field, the performance of $\mathrm{Ad}$ Hoc On-Demand Distance Vector (AODV) routing protocol 
[8] has been analysed on the basis of various quantitative performance metrics. From literature review [9-12], the comparison of simulation based studies also reveals that the use of simplistic propagation models (i.e., TRG) results in highly overestimated performance analysis. This is simply because these models do not accommodate wireless signal propagation losses that mainly happened due to reflection, diffraction, scattering, and multipaths effects (commonly caused by urban structures). Moreover in [3], the authors have discussed a modified-TRG model that accounts for signal attenuation between two nodes obstructed by a wall or building that reduce the effective signal strength received at the receiver by a random value. However this model does not cater for the increasing level of attenuation (i.e., due to increase in obstacles) among communicating nodes. In [13], the author has used the Attenuation Factor propagation model [14] in a constrained mobility environment that accounts for the number of walls between transmitter and receiver and calculates the attenuation based on rgb values (i.e., scanning the picture and calculating the colour intensity) among them. The primary objective of abovementioned work is the obstacle based mobility analysis with little attention to propagation perspective specifically with increasing obstacles. The propagation impact with varying obstacles has not been looked upon in detail. This study investigates the impact of various path loss models in an obstacle aware ad hoc environment by considering the effect of increasing obstacles (i.e., buildings, walls, etc.) on routing performance for AODV. The rest of this paper is organized as follows. Section 2 briefly summarizes the overview of AODV protocol used in our simulation analysis. Sections 3 and 4 describe the propagation loss models and mobility environment, respectively. Section 5 covers the performance metrics and Section 6 mentions the motivation and methodology adopted for this study. Section 7 contains the results and discussion about them and Section 8 summarizes the key conclusions about this study.

\section{Overview of Routing Protocol}

Due to bandwidth constraints, it is readily understandable that an on-demand approach (i.e., the route discovery is initiated only if there is a demand for it) is commonly used in wireless ad hoc network scenarios. AODV is a uniform and destination based reactive protocol. It uses a table driven routing strategy and destination sequence numbers to form an on-demand protocol. AODV maintains routing tables on the nodes. The source node initiates the route discovery process only in the presence of data packets in need of route. An intermediate node may reply with a Route Reply (RREP) only if it knows a more recent path than the one known by the sender node to the destination. A destination sequence number is used to indicate how recent the path is as follows. A new route request generated by the sender node is tagged with a higher sequence number and an intermediate node that knows the route to the destination with a smaller sequence number cannot send the RREP message. Forward links are setup when a RREP travels back along the path taken by

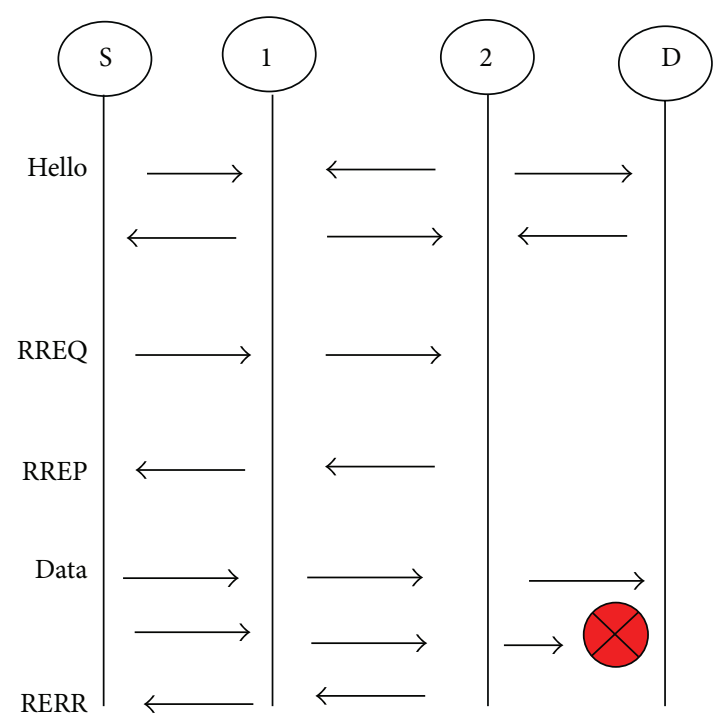

FIgURE 1: AODV routing mechanism.

Route Request (RREQ). So the routing table entries are used to forward the data packet. If an intermediate node is unable to forward the packet to the next hop or destination due to link failures, it generates the Route Error (RERR) message by tagging it with a higher destination sequence number. When the sender node receives the RERR message, it initiates a new route discovery for the destination node. Figure 1 shows the AODV mechanism implemented between a sender (S) and a destination (D) node using intermediate nodes 1 and 2 .

AODV is widely used in MANET simulations. We used this protocol with the anticipation that other routing strategies will be affected similarly due to obstacle and their impact on propagation environment (which is the focal point of this study) will not change the results significantly.

\section{Propagation Loss Models}

Radio propagation models considerably influence the performance of wireless communication networks. Radio propagation loss models are used in simulations to estimate the received signal strength of each packet received by a node. NS-2 uses the threshold values (i.e., Carrier Sense (CS_Threshold) and Receiver (RX_Threshold)), which defines the minimum possible value of the received signal strength indicator by which a node is still able to communicate successfully. If the value is smaller than the threshold, NS2 considers that the receiving node did not receive the packet successfully. The following subsection presents the deterministic and probabilistic propagation models used in our simulation scenarios.

3.1. Two-Ray Ground Path Loss Model. This model takes into consideration both direct and indirect paths between the transmitting and receiving node. This model shows better performance than free space path loss model [15] for longer distances [14]. This is an empirical model, which uses the 
following equation to calculate the approximate received power. Consider

$$
P_{r}(d)=\frac{P_{t} G_{t} G_{r} h_{t}^{2} h_{r}^{2}}{d^{4} L}
$$

where $P_{t}$ is the transmission power in watts, $G_{t}$ and $G_{r}$ are the transmitter and receiver antenna gain, $h_{t}$ and $h_{r}$ are the transmitter and receiver antenna heights, respectively, $d$ is the communication distance, and $L$ is the system loss. The TwoRay model does not give a good result for a short distance due to oscillation caused by the constructive and destructive combination of the two rays. Free space model is a better choice for smaller distances. NS-2 simulator uses a crossover distance $d_{c}$ when this model is used. If $d<d_{c}$, path loss is calculated with Friss equation and if $d>d_{c}$, TRG model is used. At the cross-over distance, both models produce the same results, so $d_{c}$ can be calculated as

$$
d_{c}=\frac{\left(4 \pi h_{t} h_{r}\right)}{\lambda}
$$

This model has been found to be reasonably accurate in predicting the large-scale signal strength over distances of several kilometres for mobile radio systems that use tall towers (i.e., height which exceeds $50 \mathrm{~m}$ ) as well as for LoS microcell channels in urban environments [16]. However, this is not a typical case in MANET scenarios (i.e., infrastructureless environment). This model is readily available in NS- 2 and was implemented by the Monarch group. We have modified this model in such a way that if there is an obstacle (i.e., a wall) existing between communicating nodes, the model subtracts the $6 \mathrm{~dB}$ power (i.e., attenuation due to single brick wall [14]) from the received signal strength. So, depending upon the number of walls that existed between two communicating nodes, modified received signal strength has been calculated from existing TRG model.

3.2. ITU LoS-NLoS Model in Street Canyons. This path loss model is recommended by ITU [6] for typical urban areas. This is a statistical model that calculates the path loss in LoS and NLoS regions and models the sharp decrease in signal strength in transition distance (i.e., going from the LoS to the NLoS region) known as the corner loss (see Figure 2).

This model was originally developed by an Ofcom project [17] based upon measurements taken in two cities (i.e., London and Reading) in U.K. This was called a "Low Height Model" with the aim of developing a model for propagation between low height terminals (see Figure 3) where both terminals are located within clutter (primarily, but not exclusively, urban and suburban clutter) [17]. Although the multihop communication scenarios were not implemented during the development of this propagation model, this model seems to be the most suitable model for MANETs (with pedestrian mobility) where nominal antenna height of transmitter and receiver is in between 1 to 1.5 meters (i.e., similar to human height).

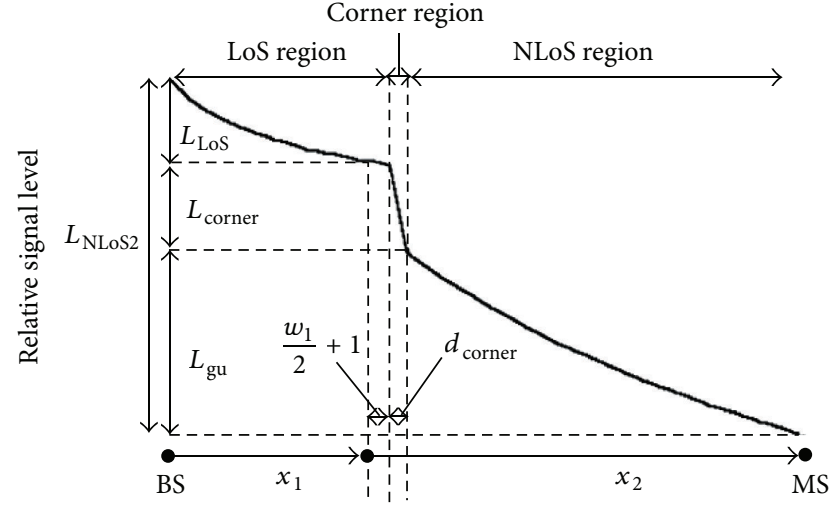

FIGURE 2: Typical trend of propagation along street canyons with low base station height for frequency range from $2 \mathrm{GHz}$ to $16 \mathrm{GHz}$ [6].

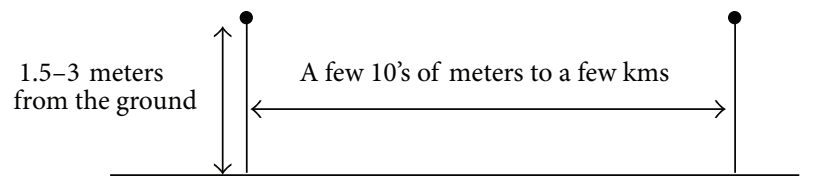

FIGURE 3: Illustration of the required ranges for height and distance between the terminals [17].

Firstly, the LoS (median) loss is calculated between $T_{x}$ and $R_{x}$ :

$$
L_{\mathrm{LoS}}^{\text {median }}(d)=32.45+20 \log _{10} f+20 \log _{10}\left(\frac{d}{1000}\right),
$$

where $d(\mathrm{~m})$ is the distance between $T_{x}$ and $R_{x}$ and $f$ $(\mathrm{MHz})$ is the operating frequency. For the required location percentage, $p(\%)$, this model calculates the LoS location correction factor by using the following Rayleigh cumulative distribution function. Consider

$$
\Delta L_{\mathrm{LoS}}(p)=1.5624 \sigma\left(\sqrt{-2 \ln \left(1-\frac{p}{100}\right)}-1.1774\right),
$$

where $\sigma$ is the standard deviation (sd) recommended as $7 \mathrm{~dB}$ through measurements. Now the total loss is calculated as

$$
L_{\mathrm{LoS}}(d, p)=L_{\mathrm{LoS}}^{\text {median }}(d)+\Delta L_{\mathrm{LoS}}(p) .
$$

The NLoS loss is calculated as

$$
L_{\mathrm{NLoS}}^{\text {median }}(d)=9.5+45 \log _{10} f+40 \log _{10}\left(\frac{d}{1000}\right)+L_{\text {urban }} \text {. }
$$

$L_{\text {urban }}$ depends upon the urban category and is $0 \mathrm{~dB}$ for suburban, $6.8 \mathrm{~dB}$ for urban, and $23 \mathrm{~dB}$ for dense urban region. The required location percentage for NLoS location correction is calculated as

$$
\Delta L_{\mathrm{NLoS}}(p)=\sigma N^{-1}\left(\frac{p}{100}\right),
$$


where $\sigma$ is recommended as $7 \mathrm{~dB}$ and $N^{-1}(\cdot)$ is the inverse normal cumulative distribution function. The total NLoS loss can be calculated as

$$
L_{\mathrm{NLoS}}(d, p)=L_{\mathrm{NLoS}}^{\text {median }}(d)+\Delta L_{\mathrm{NLoS}}(p) .
$$

For the required location percentage, $(p \%)$, the distance $d_{\text {LoS }}$ for which the LoS fraction $F_{\text {Los }}$ equals $p$ is calculated as

$$
\begin{aligned}
& d_{\text {LoS }}(p) \\
& \quad= \begin{cases}212\left[\log _{10}\left(\frac{p}{100}\right)\right]^{2}-64 \log _{10}\left(\frac{p}{100}\right) & \text { if } p<45 \\
79.2-70\left(\frac{p}{100}\right) & \text { otherwise. }\end{cases}
\end{aligned}
$$

This model suggests that if the mobile node's distance from the corner is known then $d_{\text {Los }}(p)$ is set to that distance [6].

Finally the path loss at distance $d$ is calculated by the following three conditions:

(a) if $d<d_{\mathrm{LoS}}$, then $L(d, p)=L_{\mathrm{LoS}}(d, p)$;

(b) if $d>d_{\mathrm{LoS}}+w$, then $L(d, p)=L_{\mathrm{NLOS}}(d, p)$;

(c) otherwise the loss is linearly interpolated between the following values:

$$
\begin{aligned}
L_{\mathrm{LoS}} & =L_{\mathrm{LoS}}\left(d_{\mathrm{LoS}}, p\right), \\
L_{\mathrm{NLOS}} & =L_{\mathrm{NLOS}}\left(d_{\mathrm{LoS}}+w, p\right), \\
L(d, p) & =L_{\mathrm{LoS}}+\frac{\left(L_{\mathrm{NLoS}}-L_{\mathrm{LoS}}\right)\left(d-d_{\mathrm{LoS}}\right)}{w},
\end{aligned}
$$

where width $w$ is the street width that introduces a transition region between LoS and NLoS conditions and is typically recommended as $w=20 \mathrm{~m}$ [6]. We have implemented this model into NS-2 in a hybrid way (i.e., depending upon the location of $T_{x}$ and $R_{x}$ in the simulation field, NS-2 selects appropriate path loss model).

3.3. Combined Path Loss and Shadowing Model. This model combines the simplified path loss model with shadow fading (i.e., log-normal shadowing) [7]. For this combined model, the received to transmitted power in $\mathrm{dB}$ is given as

$$
\frac{P_{r}}{P_{t}} \mathrm{~dB}=10 \log _{10} k-10 \gamma \log _{10} \frac{d}{d_{0}}-\psi_{\mathrm{dB}},
$$

where $K$ is a unitless constant that depends on the antenna characteristics and the average channel attenuation, $\gamma$ is the path loss exponent, $d_{0}$ is a reference distance for the antenna far field region, and $\psi_{\mathrm{dB}}$ is the Gauss-distributed random variable with mean zero and variance $\sigma_{\psi_{\mathrm{dB}}}$.

\section{Mobility Model}

Mobility plays an important role in network stability in MANET. Routes between communicating nodes can change rapidly due to mobility. The mobility can affect not only the communicating nodes but also the intermediate nodes and thus can have a significant influence on the network's topology and hence the performance of routing protocols. The classification of mobility and mobility models can be done on the basis of controllability and model construction [18]. In synthetic mobility models, nodes move according to a random probabilistic process whereas the trace based mobility models are based on mobility patterns that are observed experimentally. Trace based models have problems with scalability and are generally difficult to implement so synthetic models are frequently used in MANET simulations. This study implements Random Way Point (RWP) model in an obstacle aware environment. The RWP mobility model is the simplest and most widely used model for MANET studies [19].

In this mobility model, the nodes choose a random destination anywhere in the network area and start moving towards it with a velocity chosen from a speed vector $\left[0, V_{\max }\right]$. After reaching the destination, the node stops at the destination for a duration specified by the "pause time" parameter, which is the same for all nodes. All nodes repeat this process until the simulation ends. This model has some known characteristics such as nonuniform node distribution and speed decay. These characteristics have a strong influence on routing protocol performance and many variations have been suggested by researchers to cope with these issues [20]. Figure 4 shows the various scenarios used in our simulation for different sets of obstacles in the simulation field. We have used the mobility model based upon obstacles as described by [3] for this simulation work. Obstacles of various sizes with random positions in the network field have been introduced in a rectangular area of $1000 \times 600$ meters. By varying the number of obstacles in the simulation field, the performance of Ad Hoc On-Demand Distance Vector (AODV) routing protocol has been analyzed on the basis of various quantitative performance metrics.

\section{Network Performance Analysis}

The following three quantitative performance metrics are used for this study.

(1) Packet Delivery Ratio. This is the ratio of data packets successfully delivered to the number of data packets sent by the CBR sources.

(2) Normalized Routing Load. This is the ratio of the total number of routing packets generated to the number of data packets successfully delivered to destination.

(3) Mean End-to-End Delay. The delays caused by latency, buffering, queuing, retransmission, and route discovery are all included in this performance analysis; this delay is measured in milliseconds.

\section{Motivation and Methodology}

Through literature survey, it has been known that there is not any propagation model proposed yet that has been derived purely through MANETs scenario experiments. This 


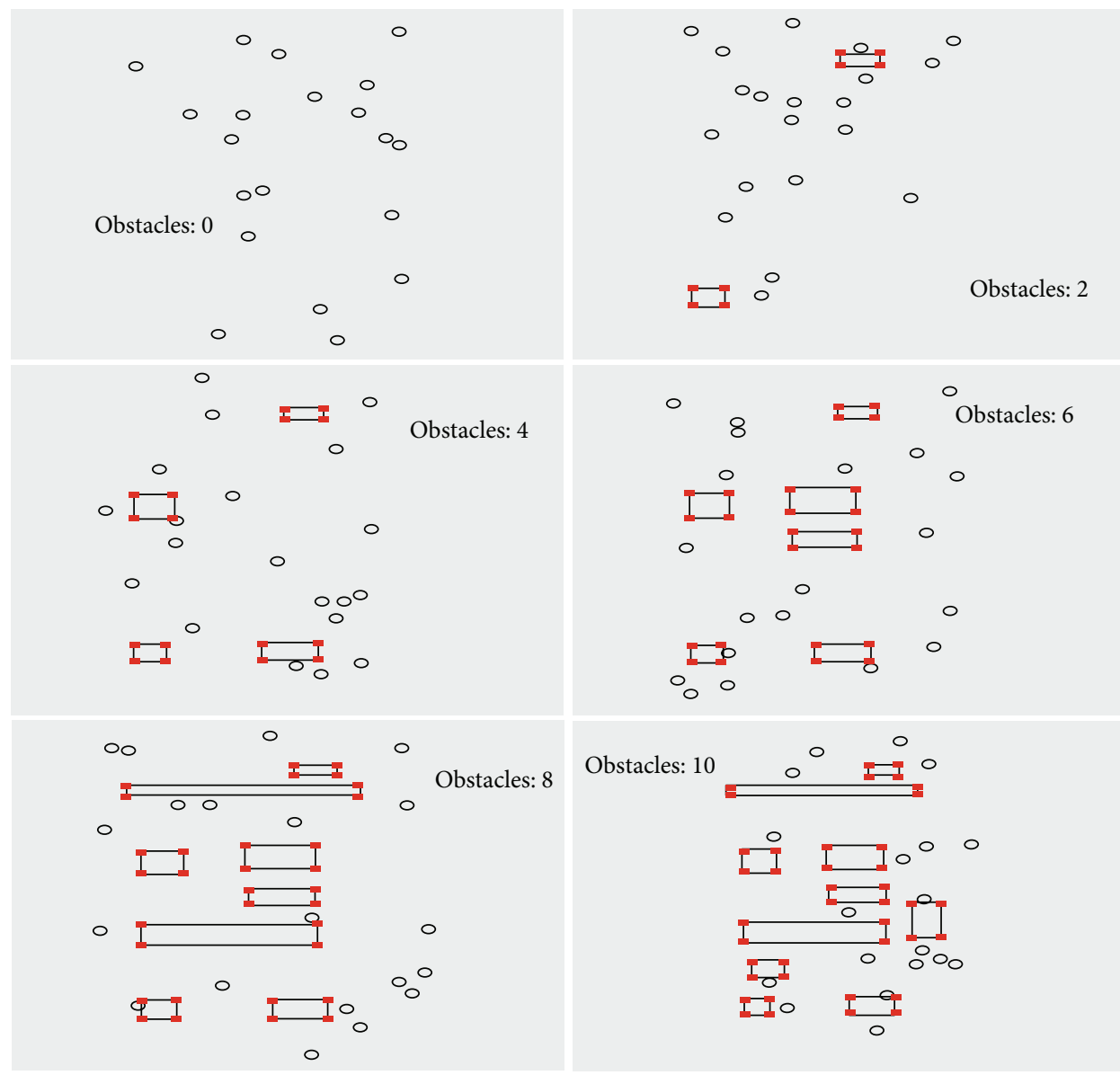

FIGURE 4: Visualization of mobility scenarios with various obstacle levels and random node movements.

is mainly because there is not any known reported test bed study done with the scope of modelling propagation environment for MANETs. Harrold et al. [21] have investigated the additional attenuation to the propagation loss effects in the cases where the antenna is very close to the ground and close to the objects such as human body (i.e., a typical case in MANETs). Green and Obaidat [22] have suggested a propagation model for ad hoc networks LoS conditions based upon signal strength measurements using WLAN traces in university campus scenario. Patwari et al. [23] have presented a path loss model for peer-to-peer communication systems based upon measurements with an antenna height of $1.7 \mathrm{~m}$ and operating frequency of $1.8 \mathrm{GHz}$ in rural and urban areas. This model considers the path loss with respect to distance and does not accommodate corner-loss effects typically found in urban NLoS cases.

Harrold and Nix [24] have shown that forming a mobileto-mobile connection (using relaying) can be useful to achieve significant benefits such as reduction in transmitted power and increase in network capacity.

Wang et al. [25] state that path loss increases with lower terminal height as does the probability of LoS. It is important to consider suitable path loss model when simulating peer-to-peer communication with low antenna height such as in MANETS. However, a generic, standalone propagation model addressing general MANET characteristics (i.e., infrastructure independent, low antenna heights, multihopping, mobility, etc.) is still a challenge for MANET research community. Wu et al. [26] have introduced an obstacle-aware mobility model in ONE (a Delay Tolerant Network (DTN) simulator) introducing obstacles of various shapes (i.e., round, hexagonal, etc.) and results have been obtained for DTN. However, the propagation model used by [26] is similar to the one mentioned in [3]. So this work covers the shortcomings in that aspect and analyses the MANET performance with the effect of increased attenuation due to increased obstacles. The selection of propagation models for our simulation work is done on the basis of relativity to the simulation environment and MANET characteristics. TRG model has been adopted according to the obstacle environment.

Furthermore, ITU models were selected as they have been developed through experimental work closely similar to the MANET environment (i.e., frequency, low antenna height, lack of tall base stations, mobility, etc.). C-Shadowing model was selected to test the MANET performance under small scale fading conditions typically found in urban environment. At this stage, it is hard to comment on the practicality of any specific model for MANET performance analysis. However, through simulation results it has been 
TABLE 1: Simulation parameters for AODV.

\begin{tabular}{lc}
\hline Parameter & Value \\
\hline Simulation time & $500 \mathrm{secs}$ \\
Area size & $1000 \times 600 \mathrm{~m}$ \\
Mean speed & $1.5 \mathrm{~m} \mathrm{sec}^{-1}$ \\
Traffic type & CBR \\
Packet size & 512 bytes \\
Connection rate & 8 pkts sec $^{-1}$ \\
Channel frequency & $2.4 \mathrm{GHz}$ \\
Mobility model & Random Way Point \\
Number of obstacles & $0,2,4,6,8,10$ \\
Transmitter power & $15 \mathrm{dBm}$ \\
$T_{x}$ and $R_{x}$ antenna gain $\left(G_{t}=G_{r}\right)$ & 1 \\
Rec. power threshold $\left(R X_{-}\right.$Threshold $)$ & $-85 \mathrm{dBm}$ \\
Carrier sense threshold $\left(C S_{-}\right.$Threshold $)$ & $-85 \mathrm{dBm}$ \\
\hline
\end{tabular}

demonstrated that the use of simplistic model for simulation may lead to false analysis. For example, in simulation based studies, packet collisions at MAC layer are calculated using received signal strength in comparison with threshold values. But the calculation of received signal strength is done by the use of propagation models in event based simulators such as NS-2. So, a node's presence in a collision domain can be falsely predicted if simple propagation models are used in simulations.

The main aim of this study is to analyze the impact of propagation loss with varying obstacle level in the simulation field. With this purpose, we generated ten mobility files for each mobility scenario. This was done in order to normalize the impact of mobility on simulation results. Each result is an average of ten simulation runs with identical input parameters but with different random seed. We used IEEE 802.11b equipped radios with Omni directional antennas (height of $1.5 \mathrm{~m}$ and with unity gain in all directions) and a receiver threshold of $-85 \mathrm{dBm}$ with a maximum transmission power of $15 \mathrm{dBm}$ at a maximum of $11 \mathrm{Mbits} / \mathrm{s}$ variable data rate. These parameters were chosen to approximate the commercially available $802.11 \mathrm{~b}$ compliant radios such as Lucent Wavelan [27].

\section{Results and Discussion}

The parameters that are used in our simulation are given in Table 1.

Figure 5 shows the PDR for different propagation loss conditions. The results indicate that all the path loss models (except TRG) show almost linear degradation in performance as the number of obstacles increases from 0 to 10 in steps of 2 . As ITU and C-Shadow models do not accommodate obstacles in their method, however increasing obstruction level means that there is more possibility of NLoS conditions among communicating nodes which increases the uses of ITU-NLoS model during simulation run and hence nodes experience higher attenuation if the channel conditions behave like ITU-NLoS environment. Furthermore, increasing obstacles decreases movement area for nodes (i.e., as nodes move only

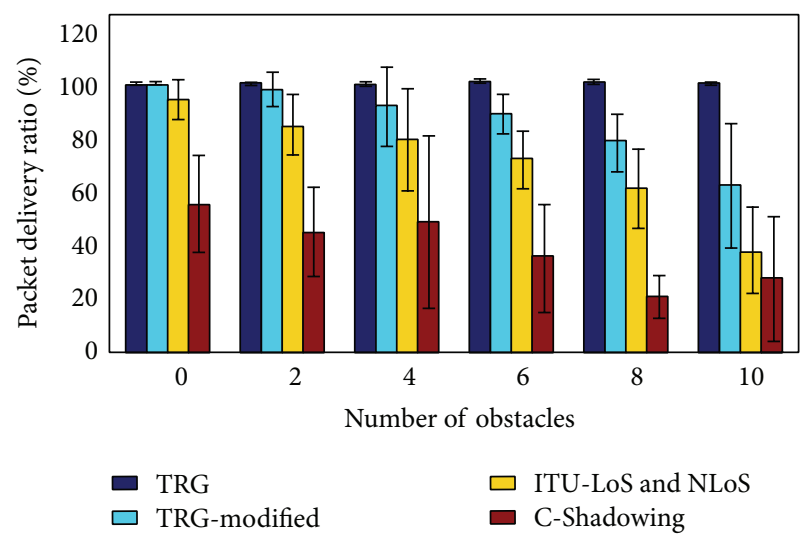

FIgURE 5: Number of obstacles versus PDR.

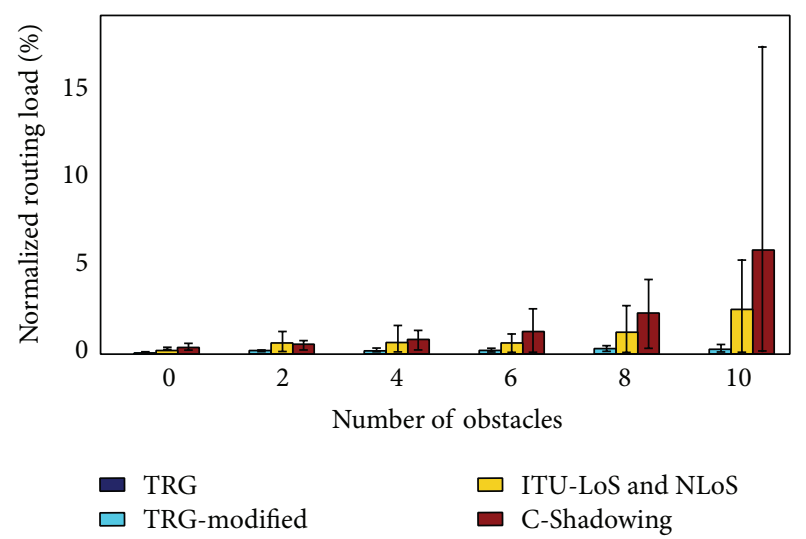

FiguRE 6: Number of obstacles versus NRL.

in available free space), which results in reduced mobility level and hence increases the possibility of more communication failures among nodes. It is worth mentioning that TRG model (although heavily used in MANETs performance analysis studies) shows almost no impact on network performance with increasing obstacles.

From Figure 6, it can be readily observed that AODV suffers with considerably higher routing load with increasing obstacles under C-Shadowing conditions. Under fading conditions such as C-Shadowing, most of the packets are dropped because interface queue is full when the transmitting node is waiting for an available route. Due to the random power fluctuations in the signal level caused by multipath propagation effects, a route found in a route discovery process may not remain a valid route that leads to more retransmission attempts and thus increases the routing load significantly.

Since we have taken the log scale on the $y$-axis as this was due to the extremely high routing load experienced with modified propagation models, hence TRG results are there but they have very small values and are kind of suppressed.

From Figure 7, it is evident that the Mean Delay is very high when the radio channel behaves like a C-Shadowing fading environment. With increasing obstacles in the simulation environment, nodes experience less connectivity due 


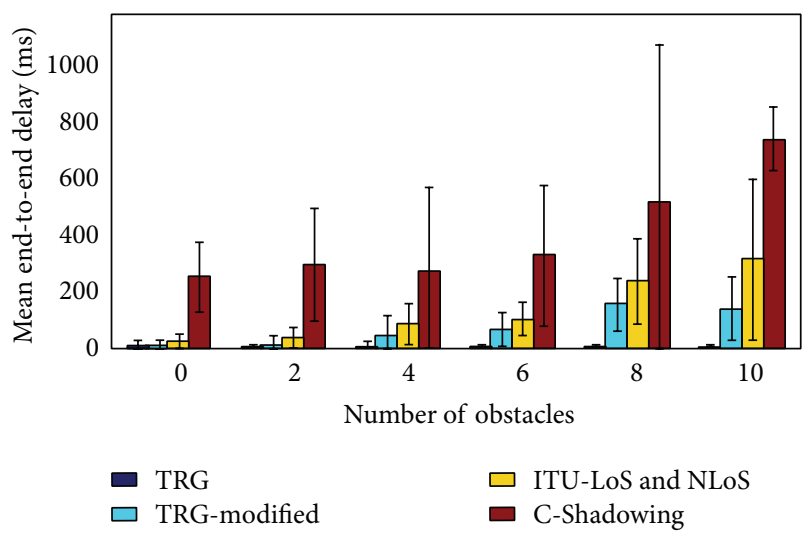

Figure 7: Number of obstacles versus Mean Delay.

to buildings, and so forth, and hence increasing routing load leads to longer communication delays.

\section{Conclusion}

This study analyses the impact of various propagation loss models on the performance of AODV in an obstructed environment. Two new propagation models have been added into NS-2 simulator and the existing Two-Ray Ground propagation model has been modified in order to accommodate increasing level of attenuation due to increase in obstacle. Results indicate that the network performance is affected with increase in obstacles. AODV suffers from low PDR, higher NRL, and Mean Delay if the communication channel behaves like C-Shadowing ITU or modified-TRG models in comparison with TRG model. This is mainly because of the fact that obstacle levels are not considered by TRG model. Moreover, ITU model incorporates fading margins for LoS/NLoS scenarios and hence degrades received signal strength. This study verifies that underestimating physical layer in MANETs will lead to more optimistic rather than realistic network performance analysis. Furthermore, it is hard to identify any particular propagation model more suitable for MANET performance analysis. However, it can be said that use of simple propagation model such as TRG may lead to overly optimistic network performance. In future, this study will lead to the investigation of propagation effects in MANETs based upon test bed work using ray tracing algorithms.

\section{Conflict of Interests}

The authors declare that there is no conflict of interests regarding the publication of this paper.

\section{Acknowledgments}

This research was supported by Basic Science Research Program through the National Research Foundation of Korea (NRF) funded by the Ministry of Education (2013R1A1A2061978). It was also funded by Research Fund,
Bahria University, Islamabad. This research was also funded by the MSIP (Ministry of Science, ICT and Future Planning), Korea, in the Human-Care Contents Development Program 2014.

\section{References}

[1] “The Network Simulator ns-2," version ns-2.34, http://www.isi .edu/nsnam/ns/doc.

[2] S. Kurkowski, T. Camp, and M. Colagrosso, "MANET simulation studies: the incredibles," ACM SIGMOBILE Mobile Computing and Communications Review, vol. 9, no. 4, pp. 5061, 2005.

[3] A. P. Jardosh, E. M. Belding-Royer, K. C. Almeroth, and S. Suri, "Real-world environment models for mobile network evaluation," IEEE Journal on Selected Areas in Communications, vol. 23, no. 3, pp. 622-632, 2005.

[4] C. Papageorgiou, K. Birkos, T. Dagiuklas, and S. Kotsopoulos, "Simulating mission critical mobile ad hoc networks," in Proceedings of the 4th ACM International Workshop on Performance Monitoring, Measurement, and Evaluation of Heterogeneous Wireless and Wired Networks (PM2HW2N '09), pp. 143-150, Tenerife, Spain, October 2009.

[5] L. F. Zang and G. B. Rowe, "Improved modelling for mobile Adhoc networks," IEEE Electronics Letters, vol. 43, no. 21, pp. 11561157, 2007.

[6] ITU-R P.1411-5, "Propagation data \& Prediction methods for the planning of short range outdoor radio communication systems \& radio local area networks in the frequency range of $300 \mathrm{MHz}$ to $100 \mathrm{GHz}, 2009$.

[7] A. Goldsmith, Wireless Communications, Cambridge University Press, 2005.

[8] E. Perkins, B. Royer, and S. Das, "Ad hoc on-demand distance vector (AODV) routing," Tech. Rep. RFC 3561, 2003.

[9] S. Agrawal, S. Jain, S. Sharma, and R. Gupta, "Mobility based performance analysis of AODV and DYMO under varying degree of node misbehavior," International Journal of Computer Applications, vol. 29, no. 7, pp. 36-41, 2011.

[10] A. Singh, A. Könsgen, and C. Goerg, "Enhancing AODV performance by improved link metrics," in Proceedings of the 5th International Conference on Information and Automation for Sustainability (ICIAfS '10), pp. 183-188, Colombo, Sri Lanka, December 2010.

[11] C. P. Agrawal, M. K. Tiwari, and O. P. Vyas, "Evaluation of AODV protocol for varying mobility models of MANET for ubiquitous computing," in Proceedings of the 3rd International Conference on Convergence and Hybrid Information Technology (ICCIT '08), vol. 1, pp. 769-774, November 2008.

[12] A. K. Gupta, H. Sadawarti, and A. K. Verma, "Performance analysis of AODV, DSR \& TORA routing protocols," IACSIT International Journal of Engineering and Technology, vol. 2, no. 2, 2010.

[13] H. A. Lagar-Cavilla, G. Baron, T. E. Hart, L. Litty, and E. de Lara, "Simplified simulation models for indoor MANET evaluation are not robust," in Proceedings of the 1st IEEE Communications Society Conference on Sensor and Ad Hoc Communications and Networks (SECON '04), pp. 610-620, Santa Clara, Calif, USA, October 2004.

[14] T. S. Rappaport, Wireless Communications, Principles and Practice, Prentice, 1996. 
[15] H. T. Friis, "A note on a simple transmission formula," Proceedings of the IRE, vol. 34, no. 5, pp. 254-256, 1946.

[16] M. J. Feuerstein, K. L. Blackard, T. S. Rappaport, S. Y. Seidel, and H. H. Xia, "Path loss, delay spread, and outage models as functions of antenna height for microcellular system design," IEEE Transactions on Vehicular Technology, vol. 43, no. 3, pp. 487-497, 1994.

[17] 2007, http://stakeholders.ofcom.org.uk/binaries/research/ technology-research/finalreportph2.pdf.

[18] W.-J. Hsu, T. Spyropoulos, K. Psounis, and A. Helmy, "Modeling spatial and temporal dependencies of user mobility in wireless mobile networks," IEEE/ACM Transactions on Networking, vol. 17, no. 5, pp. 1564-1577, 2009.

[19] J. Broch, D. A. Maltz, D. B. Johnson, Y. Hu, and J. Jetcheva, "A Performance comparison of multi-hop wireless ad hoc network routing protocols," in Proceedings of the 4th Annual ACM/IEEE international Conference on Mobile Computing and Networking (Mobicom '98), pp. 85-97, July 1998.

[20] T. Camp, J. Boleng, and V. Davies, "A survey of mobility models for ad hoc network research," Wireless Communications and Mobile Computing, vol. 2, no. 5, pp. 483-502, 2002.

[21] T. J. Harrold, A. R. Nix, and M. A. Beach, "Propagation studies for mobile-to-mobile communications," in Proceedings of the IEEE 54th Vehicular Technology Conference (VTC'01), vol. 3, pp. 1251-1255, October 2001.

[22] D. B. Green and M. S. Obaidat, "An accurate line of sight propagation performance model for ad-hoc 802.11 wireless LAN (WLAN) devices," in Proceedings of IEEE International Conference on Communications (ICC '02), vol. 5, pp. 3424-3428, New York, NY, USA, April 2002.

[23] N. Patwari, G. D. Durgin, T. S. Rappaport, and R. J. Boyle, "Peerto-peer low antenna outdoor radio wave propagation at 1.8 GHz," in Proceedings of the IEEE VTS 50th Vehicular Technology Conference (VTC '99), vol. 1, pp. 371-375, IEEE, Houston, Tex, USA, September 1999.

[24] T. J. Harrold and A. R. Nix, "Intelligent relaying for future personal communication systems," in Proceedings of the IEE Colloquium on Capacity and Range Enhancement Techniques for the Third Generation Mobile Communications and Beyond, Ref. no. 2000/003, pp. 9/1-9/5, 2000.

[25] Z. Wang, E. K. Tameh, and A. R. Nix, "Joint shadowing process in urban peer-to-peer radio channels," IEEE Transactions on Vehicular Technology, vol. 57, no. 1, pp. 52-64, 2008.

[26] S. L. Wu, C. Y. Lin, Y. C. Tseng, and J. L. Sheu, "A new multichannel MAC protocol with on-demand channel assignment for multi-hop mobile ad hoc networks," in Proceedings of the International Symposium on Parallel Architectures, Algorithms and Networks (I-SPAN '00), pp. 232-237, Dallas, Tex, USA, December 2000.

[27] B. Tuch, "Development of WaveLAN, an ISM band wireless LAN," AT\&T Technical Journal, vol. 72, no. 4, pp. 27-33, 1993. 

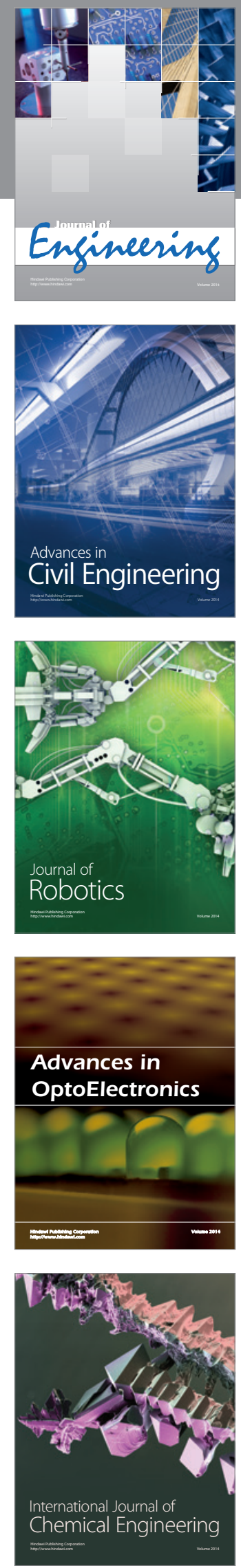

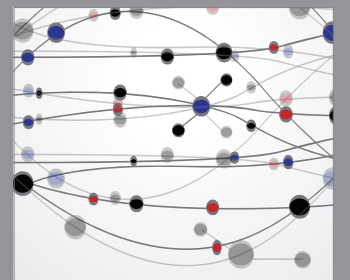

The Scientific World Journal
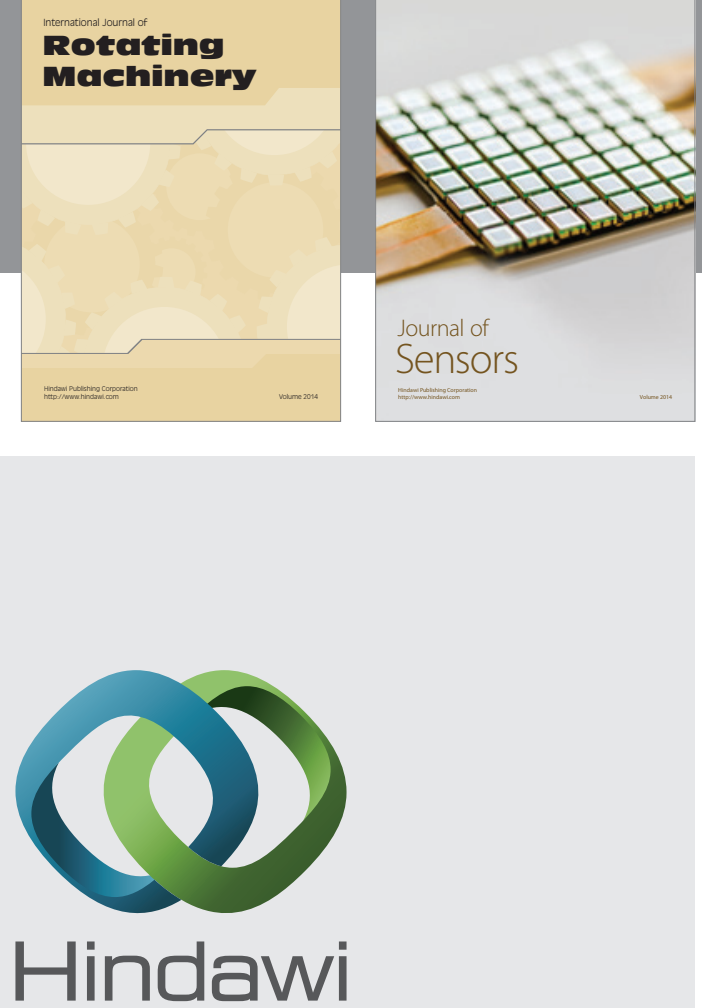

Submit your manuscripts at http://www.hindawi.com
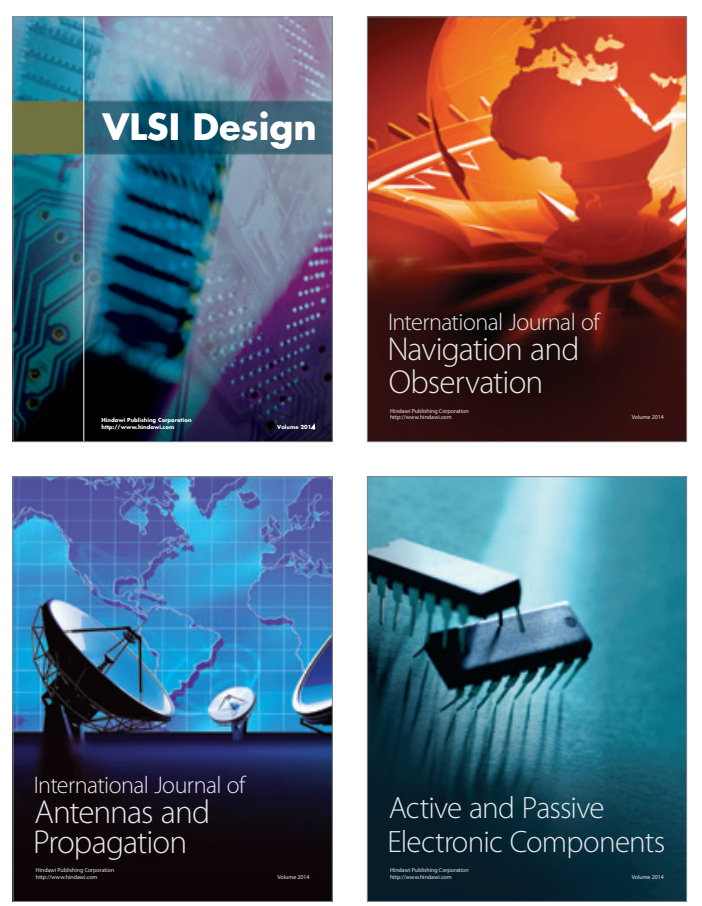
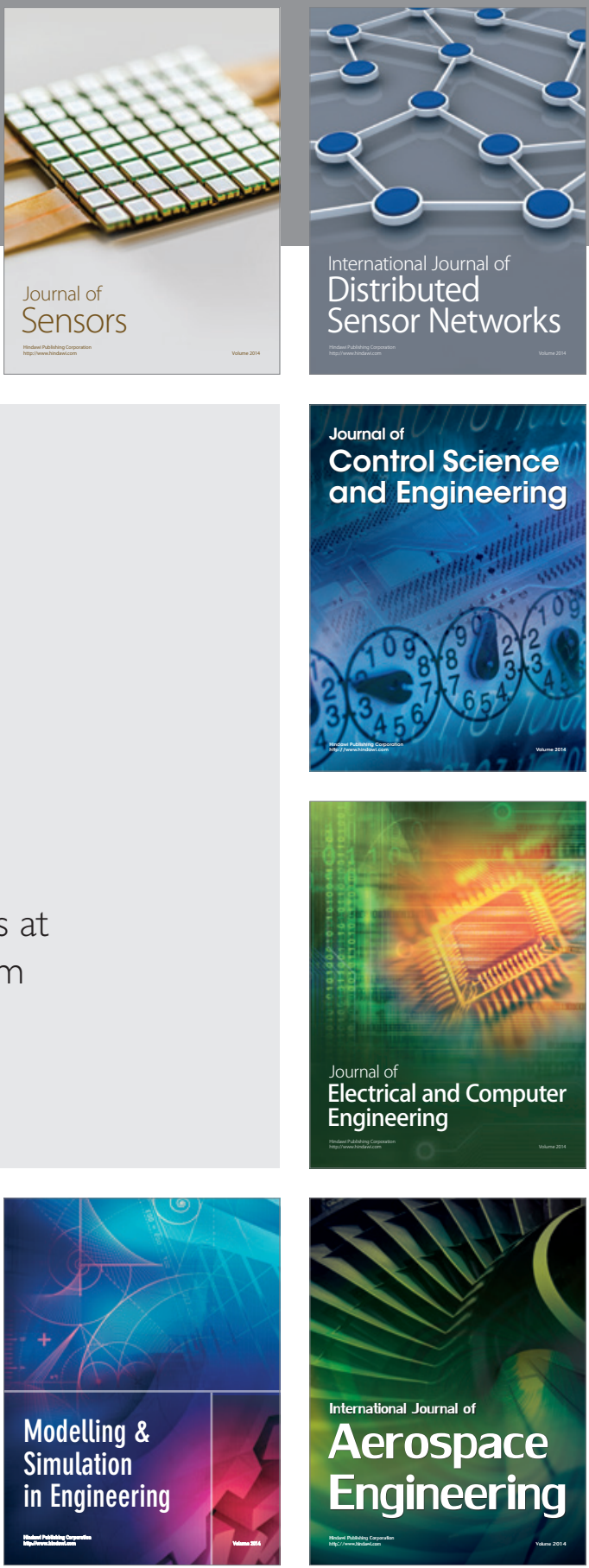

Journal of

Control Science

and Engineering
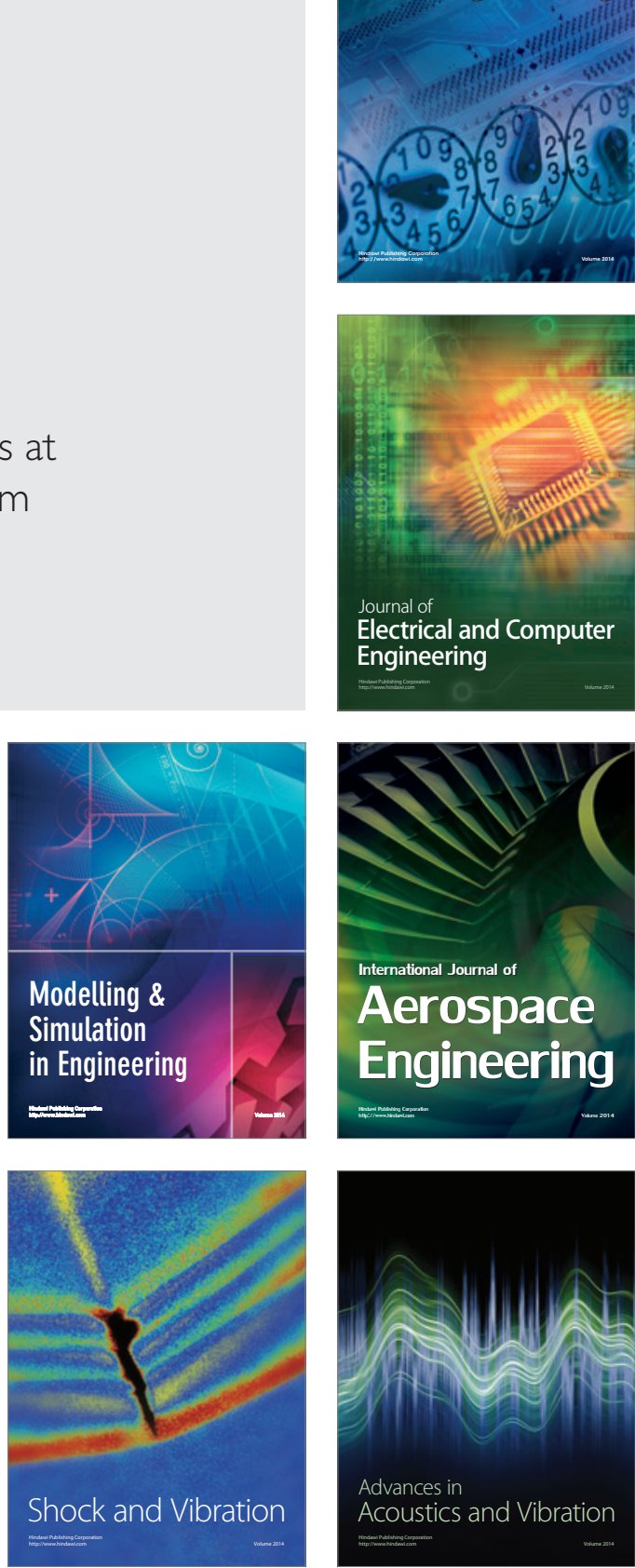